\title{
METHOD FOR INCREASING THE EFFICIENCY OF AUTOMATIC FIRE EXTINGUISH SYSTEM AT OBJECTS OF POWER
}

\author{
Margarita Dmitrienko ${ }^{1, a}$ \\ ${ }^{1}$ National Research Tomsk Polytechnic University, 634050 Tomsk, Russia
}

\begin{abstract}
Operation of energy facilities requires compliance with all safety standards, and especially fire safety. Emergency situations that arise when operated the power equipment damage not only the objects of the technosphere but also the environment. In recent years, can be noted a trend of quite intensive development of technological bases of technology water mist fire extinguishing. Using the methods of optical panoramic imaging PIV, IPI and the method of high-speed video recording were performed the experimental studies of the characteristics of evaporation of large single water droplets as they pass through the flames of oil and oil products with varying parameters of the processes (the initial size of 2-6 $\mathrm{mm}$, the rate of $2-4 \mathrm{~m} / \mathrm{s}$ and the temperature of water drops $290-300 \mathrm{~K}$, the temperature of the combustion products 185-2073 K). Was established decisive influence droplet size, velocities at which droplets enter the gaseous medium, the initial water temperature on heating rate and evaporation of droplets in a stream of high-temperature combustion products.
\end{abstract}

\section{Introduction}

Fire extinguishing systems are the most promising, as water reserves in the world are practically inexhaustible. In addition, the water has a high fire-extinguishing efficiency due to cooling and phlegmatizing effects on the seat of fire. However, the relatively low ability of water to wetting the burning solid surfaces, the formation of stable barriers on the surface of the flammable liquid and the penetration into closed areas is reduces the effectiveness of its application [1-5]. As a result, water flow for fire fighting reaches significant values. Therefore, one of the main challenges of founders the modern fire extinguishing systems is increase the efficiency of water use [6].

The purpose of this work - the study of the evaporation of large single water droplets as it passes through the flames of oil and oil products with varying parameters of the processes (the initial size of 2-6 mm, the rate of 2-4 m/s and the temperature of the water droplets 290-300 K, the temperature of the combustion products $185-2073 \mathrm{~K}$ ).

a Corresponding author : mad2@tpu.ru

This is an Open Access article distributed under the terms of the Creative Commons Attribution License 4.0, which permits unrestricted use, distribution, and reproduction in any medium, provided the original work is properly cited. 


\section{Experimental setup and study technique}

A scheme of our experimental setup is shown on fig. 1. The main elements of the installation are: high-speed video camera 1 (the frame rate at least 3000 frames per second) and the cross-correlation video camera 2 (resolution - $2048 \times 2048$ pixels, frame rate $-1.5 \mathrm{~Hz}$ ).

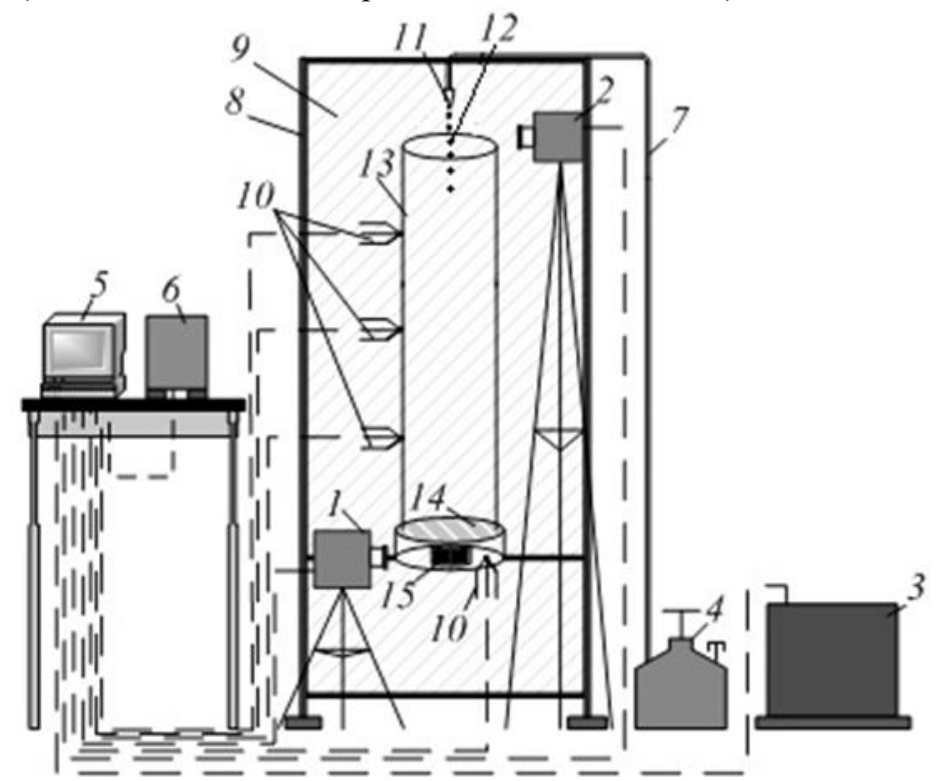

Figure 1. Scheme of an experimental setup: 1 - high-speed video camera; 2 - cross-correlation video camera; 3 heating device; 4 - water tank; 5 - personal computer; 6 - multichannel technological registrar; 7 - water supply tubing; 8 -aluminum rack; 9 - glass wall of the chamber; 10 - thermocouples; 11 -dosing devise; 12 - water droplets; 13 - quartz tube; 14 - hollow tube; 15 - flammable liquid

Under identical initial conditions was carried out at least 10 series of experiments. The tank 4 is filled with water. The control of temperature of water was carried out using chromel-copel thermocouple, which was install into a tank 4 . The tank 4 connected with dosing device 11 , which was arranged according to the required parameters of the water droplets. The initial temperature of the water droplets is maintained using a heating device 3 . The flow of high temperature gas formed in the cylindrical channel 13 (height $-1 \mathrm{~m}$, diameter $-0.3 \mathrm{~m}$ ). At the base of the channel 13 installed a hollow cylinder 14 (height $-0.1 \mathrm{~m}$, inner diameters $-0.26 \mathrm{~m}$ and outer diameters $-0.3 \mathrm{~m}$ ), in which located the fire source 15. The temperature of the gases (combustion products) is measured using three tungsten-rhenium thermocouples 10 (range of temperature measurement $0 \div 2473 \mathrm{~K}$, the error of \pm $282 \mathrm{~K})$.

To measure the velocity of the gases used panoramic optical method PIV [7]. For this purpose into the gaseous medium injected "tracers" - titanium dioxide particles. The velocity of "tracers" was about $1.5 \mathrm{~m} / \mathrm{s}$. Then on the workstation 5 to run special software "ActualFlow", turned on the dosing device 11 and carried out the procedure of registration videograms. The dimensions of each video frame in the gaseous medium is selected in accordance with a characteristic length of recorded trajectories of investigated colliding droplets. Dimensions of water droplets $\mathrm{R}_{\mathrm{m}}$ and theirs velocities $\mathrm{U}_{\mathrm{m}}$ measured using optical panoramic methods PIV and IPI $[8,9]$.

\section{Results and discussion}

Fig. 2 shows the experimental dependencies of the fraction of water evaporated $\Delta R$ from the initial droplet size $R_{\mathrm{d}}$ when moving in the flames of oil and oil products. These experimental dependencies show that when the initial droplet size $R_{\mathrm{d}}$ is increased then the parameter $\Delta R$ is decreased. This can be 
explained by the increase of their warm-up time of the surface layer to a temperature of the beginning of intensive evaporation. These conclusions can be made for all combustion products of flammable liquids used in the experiments.

Fig. 3 shows the experimental dependencies of the fraction of water evaporated $\Delta R$ from the initial velocity $U_{\mathrm{d}}$ of the water droplets when moving in the flames of oil and oil products at. Analysis of the dependencies can be concluded that when the initial velocity of the drops is increased then the parameter $\Delta R$ is decreased.

Fig. 4 shows the experimental dependencies of the fraction of water evaporated $\Delta R$ from the starting water temperature $T_{\mathrm{w}}$. The water temperature varied in the range of $290 \mathrm{~K}$ to $350 \mathrm{~K}$. From the experimental dependencies it is evident that the higher the initial temperature of the water droplets generated, the more intensive the process of evaporation.

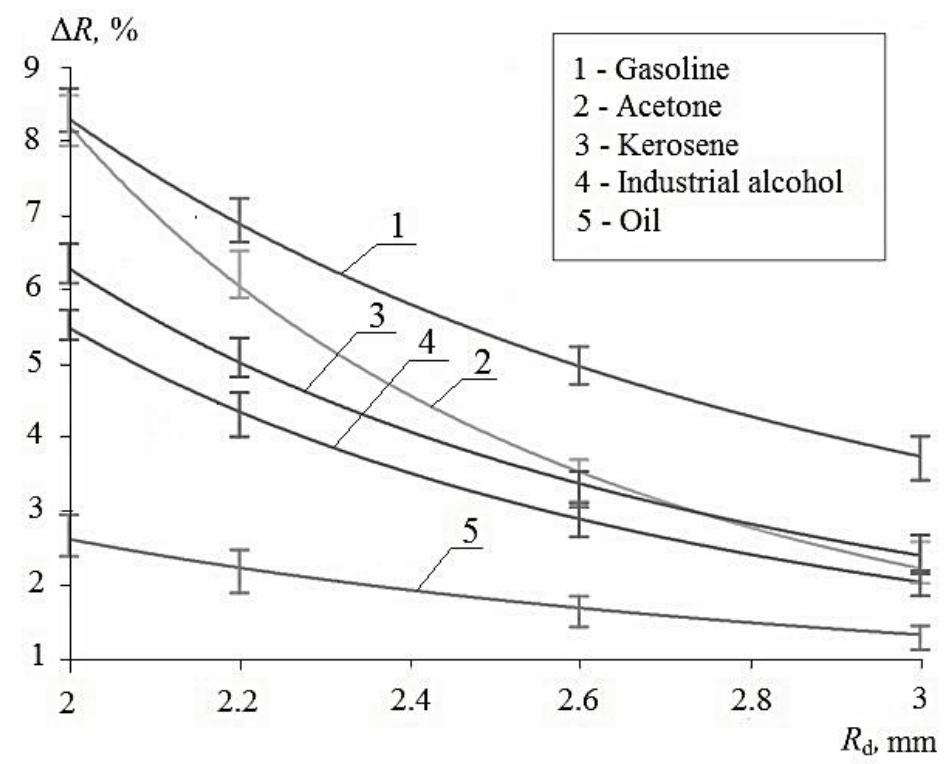

Figure 2. Experimental dependencies of the fraction of water evaporated $\Delta R$ from the initial water droplet size $R_{\mathrm{d}}$ $\left(U_{\mathrm{d}}=3.5 \mathrm{~m} / \mathrm{s}\right)$

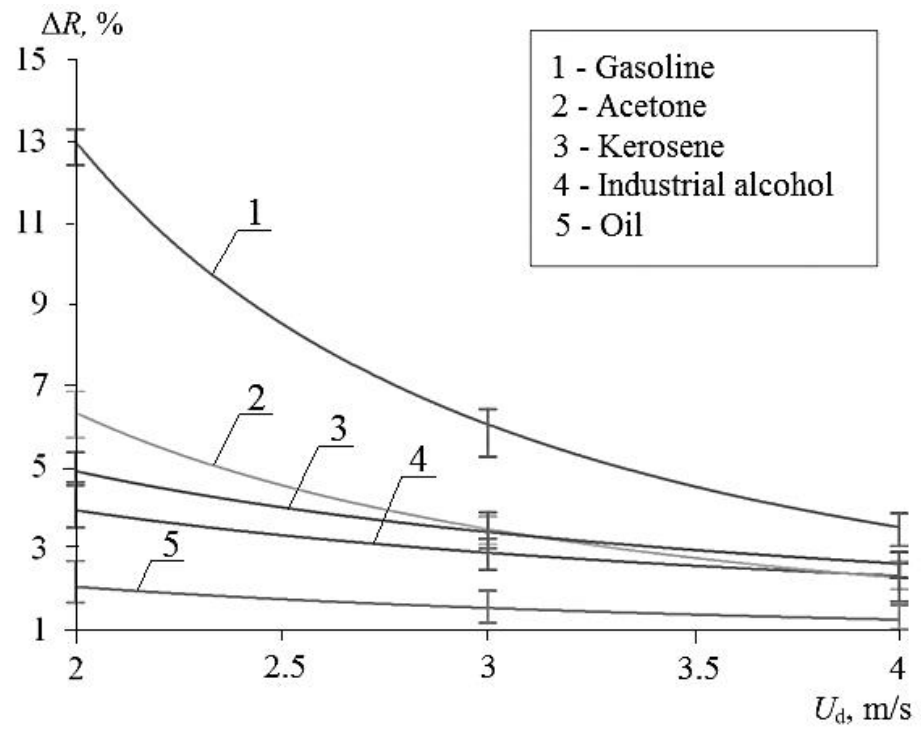


Figure 3. Experimental dependencies of the fraction of water evaporated $\Delta R$ from the initial velocity of the water droplets $U_{\mathrm{d}}\left(R_{\mathrm{d}}=2.8 \mathrm{~mm}\right)$

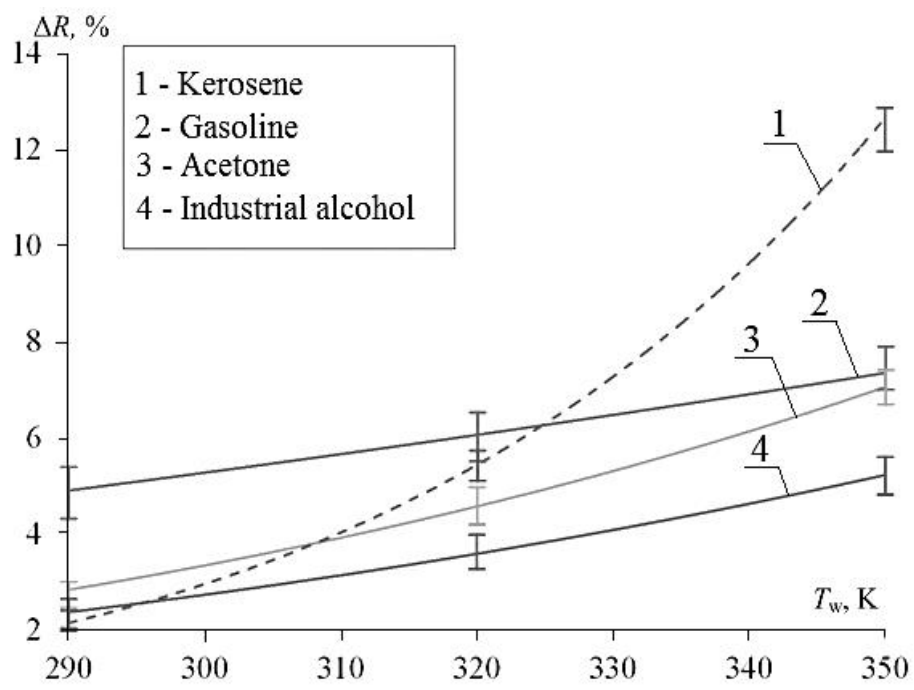

Figure 4. Experimental dependencies of the fraction of water evaporated $\Delta R$ from the starting water temperature $T_{\mathrm{w}}\left(R_{\mathrm{d}} \approx 2.7 \mathrm{~mm}\right)$

\section{Conclusion}

Experimental researches of evaporation of large single water droplets it passes through the flames of oil and oil products using optical methods (PIV, IPI) and the high-speed video recording method showed that a decisive influence on the rate of heating and evaporation have a droplet size, droplet velocity and the initial temperature of water. The smaller droplet size and speed, the greater the intensity of evaporation. In addition, the higher temperature of water droplets, the more intense the evaporation is.

The study was financially supported by the grant of Russian Foundation for Basis Research (project 15-38-20006).

\section{References}

1. B. Yao, B.H. Cong, J. Qin, W.K. Chow. Fire Saf. J. 47, 32 (2012)

2. $\mathrm{Na}$ Meng, Longhua $\mathrm{Hu}$, Shuai Liu, Long Wu, Longfei Chen, Binghai Liu. J. Fire Sci. 30, 138 (2012)

3. M. Guptaa, R. Rajoraa, S. Sahaia, R. Shankar, A. Ray, S.R. Kale, Fire Safety Journal 54, 130 (2012)

4. R.S. Volkov, G.V. Kuznetsov, P.A. Strizhak, J. Eng. Phys.Thermophys. 87, 450 (2014)

5. R.S. Volkov, O.V. Vysokomornaya, G.V. Kuznetsov, P.A. Strizhak, J., Eng. Phys.Thermophys. 86, $1413(2013)$

6. Karpov A. I., Novozhilov V. B., Galat A. A., Bulgakov V. K. Fire Saf. Sci. 27, 753 (2005)

7. J.V. Simo Tala, S. Russeil, D. Bougeard, J.-L. Exp. Therm. and Fluid Sci. 50, 45 (2013)

8. T. Kawaguchi, Y. Akasaka, M. Maeda, Measurement Science and Technology 13, 308 (2002)

9. D. Damiani, E. Meillot, D. Tarlet, J. Therm. Spray Technol. 23, 340 (2014) 